A Comparison of Standard Evasion

Scenarios at Near Regional Distances
R. Bos
F. App
E. Jones
T. Dey
J. Kamm

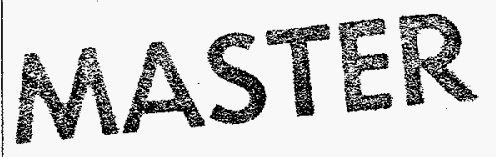




\section{DISCLAIMER}

Portions of this document may be illegible in electronic image products. Images are produced from the best available original document. 


\title{
A Comparison of Standard Evasion Scenarios at Near Regional Distances
}

by

R. Bos, F. App, E. Jones, T. Dey, and J. Kamm

\begin{abstract}
We performed numerical simulations of three nuclear testing evasion scenarios. These calculations were done in two parts. The first part was done near source (within $10 \mathrm{~km}$ ) with a non-linear weapons effects code. Particle velocity histories from the non-linear code were linked to an elastic linear finite-difference code for the second part. Seismic waveforms from the evasion scenario calculations were compared with the waveforms for a non-evasive explosion calculation at near regional distances. The results of this comparison suggest that it may be important to include realistic stratigraphy in such simulations: the overall wave amplitude in the present simulations is reduced by only a factor of 3-5 in contradiction to factors of 20-100 in "classical" decoupling analyses for ideal (i.e., unlayered) media. Two of the evasion scenarios simulated retain explosive waveform characteristics at near regional distances, while the third scenario indicates that certain source geometries might lead to more nonexplosive (i.e., earthquake-like) seismic signals.
\end{abstract}

\subsection{Introduction}

Potential nuclear test detection evasion scenarios have been studied for some time (Latter et al. 1961). Questions about evasion scenarios and the ability to detect nuclear tests conducted evasively have taken on new meaning with the possible entry into force of a Comprehensive Test Ban Treaty (CTBT), which may prohibit all testing of nuclear devices. Even without the CTBT raising the issue of detection, from a national security viewpoint it is critical to be able to determine whether a potential proliferant is actually attempting to test the design and development of nuclear weapons.

Many calculations have been done on various evasion scenarios (Terhune et al. 1979, Stevens et al. 1991, Glenn 1993, Dey 1993, Dey \& Bos 1995a) and some experiments have been done in the US (Springer et al. 1968, Murphy \& Barker 1994, Glenn 1995, Reinke 1995) and by the Russians (Murphy et al. 1994, Spivak \& Adushkin 1995). Most calculational and some close-in experimental results seem to indicate a reduction in signal of a factor of 10 to 100 , depending on the specific investigation. To the authors' knowledge, there has not been a study published of experimental results at regional distances from an evasion test. Most available results are based on simulations from what we will term "strong motion codes" which incorporate non-linear effects for near source simulations of 
high energy density sources like nuclear tests or large high explosive (HE) tests. These codes were primarily developed and used for weapons effects and containment studies. The results from this type of code show in general very good agreement with experiments out to 1 scaled $\mathrm{km}$ from the source (e.g., the Non-Proliferation Experiment (NPE) simulation contained in Kamm \& Bos 1995). As a class, the strong motion codes at present do not provide accurate simulations of the wave propagation out to regional distances. Typically this is due to equation of state inadequacies for very late times $(10-100 \mathrm{~s})$ or inability to handle the numerics of the small velocities and stresses that exist at distances greater than 10 scaled $\mathrm{km}$ from the source. In order to extrapolate results simulated at 1 to 10 scaled $\mathrm{km}$ from the source out to regional distances, an assumption generally is made that only the low frequency component of the wave will survive out to regional distances. In most cases, since the calculation is already far into the elastic material response regime, this assumption would seem to be justified. Under this assumption, the reduced velocity potential (RVP) spectrum is typically used as a figure of merit in estimating the decoupling factor of the evasion scenario being studied. The decoupling factor is a ratio of the lowest frequency component of the RVP spectrum obtained from an evasion scenario simulation to the lowest frequency component of a tamped nuclear explosion simulation of identical source yield. A tamped explosion is one in which the source energy is deposited directly into the surrounding geologic material, with no voids, gaps, or other energy modifying structures. Most actual underground nuclear tests were conducted in tamped conditions. The decoupling factor derived from RVP spectra is then used to predict the ratio of signal strengths at regional distance from the source.

While signal reduction is important in the CTBT context, this report addresses a different question. We will address the degree to which the seismic signal from a standard evasion scenario at a near regional distance from the source differs from the seismic signal from a non-evasive fully tamped explosive source with the same yield at the same distance from the source. Although amplitude reduction may make detection difficult, it is important to know the extent to which the signal from the evasive scenario maintains the explosive characteristics of a tamped explosion.

Using recently developed linking capabilities between the non-linear strong motion codes and the linear elastic $4^{\text {th }}$-order finite-difference code AFD (Kamm et al. 1996), we can now explore directly the signal generated by a simulation of an evasion scenario at regional distances with full non-linear, near source behavior taken into account. This work compares three potential evasion scenarios to a standard tamped nuclear explosion. Synthetic seismograms are obtained at near regional distances of $100 \mathrm{~km}$ and $200 \mathrm{~km}$ from the source.

\subsection{Description of the Simulations}

Three evasion scenarios were chosen for comparison with a tamped case. These are (1) a spherical cavity of $32 \mathrm{~m}$ radius, (2) a mine chamber of radius $32 \mathrm{~m}$ and a height of $3 \mathrm{~m}$ (a "tuna can" shaped chamber), and (3) a rubblized chamber of radius $32 \mathrm{~m}$ and height $32 \mathrm{~m}$; these scenarios are described in more detail below. The spherical cavity, while not the easiest structure to mine, is the evasion scenario most commonly studied. The mine chamber is a readily available type of underground mining room and is representative of a class of mined rooms typically produced in everyday mining activities. The rubble-filled chamber is representative of another standard mining technique and was the focus of a previous study by some of the authors (Dey \& Bos 1995a). 
All the calculations were done in exactly the same manner. The initial strong motion calculation used SMC123 (Dey \& Kamm 1994) in its cylindrical (r-z) mode (see Section 3.0 for the code description). The energy source had a energy yield of $1 \mathrm{kt}$ at depth $300 \mathrm{~m}$ from the surface. The initial (and only) layer in the SMC run was comprised of granite. Granite and the depth of burial were chosen to assure containment of the resultant high pressure cavity gases. Sizes of the cavities were based on reasonable mining practices and scaled decoupling factors for a spherical cavity of radius $32 \mathrm{~m}$. Particle velocity information was obtained along a boundary $1 \mathrm{~km}$ from the source to be used for linking to the AFD elastic finite-difference code (Kamm et al. 1996).

Each of the calculations was then linked to AFD using the same $\mathrm{r}-\mathrm{z}$ cylindrical mode employed in the SMC123 calculations. The AFD simulations had five geologic layers obtained from the standard IASP91 crustal velocity model (see Table 1). The calculation was run out to $200 \mathrm{~s}$ and velocity histories were obtained at points on the surface at $100 \mathrm{~km}$ and $200 \mathrm{~km}$ from the source. The final mesh was $320 \times 160 \mathrm{~km}$ with $160 \mathrm{~m}$ square cells, resulting in a frequency resolution of between 3 and $4 \mathrm{~Hz}$. Anelasticity (seismic Q) was not included in the calculation. The final cell size was chosen to support as much of the $\mathrm{P}$ and $\mathrm{S}$ waveform as possible while still small enough to run in memory. This cell size, however, was not small enough to stop numerical ringing from developing for the surface wave, which typically requires higher frequencies to adequately describe the waveform than do the $\mathrm{P}$ and $\mathrm{S}$ waves. The non-physical ringing feature is particularly evident when linking from a strong motion code where the surface waveform is reliably described at higher frequencies. This ringing is evident in Figure 1 in the waveforms after $35 \mathrm{~s}$. The cause and effect of this numerical ringing is described in "User's Guide to AFD v. 1.0" (Kamm et al. 1996). This effect only occurs in simulations of idealized layered materials where the surface wave can remain coherent.

Table 1

Crustal model velocities and densities used in the calculations described in the text.

\begin{tabular}{|c|c|c|c|c|}
\hline Layer & Depth $(\mathrm{km})$ & $\mathfrak{G}(\mathrm{m} / \mathrm{s})$ & $\mathcal{c}_{\mathrm{s}}(\mathrm{m} / \mathrm{s})$ & $\rho\left(\mathrm{g} / \mathrm{cm}^{3}\right)$ \\
\hline 1 & $0-20$ & 5444 & 3009 & 2.670 \\
\hline 2 & $20-36$ & 6500 & 3750 & 2.900 \\
\hline 3 & $36-80$ & 8040 & 4471 & 3.379 \\
\hline 4 & $80-120$ & 8048 & 4494 & 3.372 \\
\hline 5 & $120-160$ & 8120 & 4505 & 3.368 \\
\hline
\end{tabular}

\subsection{Tamped Explosion}

In this simulation, the energy was initially deposited in a $1 \mathrm{~m}$ radius spherical volume in the granite. This type of calculation simulates a generic "tamped" nuclear explosion which is contained on all sides by geologic material. This calculation is used as the baseline for comparison with the other explosive driven simulations. 


\subsection{Spherical Cavity}

A spherical open volume of radius $32 \mathrm{~m}$ is loaded with the $1 \mathrm{kt}$ energy evenly distributed over the volume. This initial condition is what would obtain in a cavity at the time when the hydrodynamic shock wave hits the cavity walls. This approximation ignores the initial radiation-induced shock wave produced at the cavity walls. In more detailed calculations (Stevens et al. 1990), the radiation effects are found to be negligible.

\subsection{Cylindrically Shaped Mine Room}

A tuna can shaped mine room of radius $32 \mathrm{~m}$ and height $3 \mathrm{~m}$ is filled with $1 \mathrm{kt}$ of energy. The same assumptions of energy distribution as in the spherical cavity were made here. In this case, it should be noted that the assumption of a uniform energy density is less accurate. The positioning of the source would induce a shock in the ceiling and floor of the chamber immediately above and below the source, producing a ellipsoidal shaped shock wave emanating from the chamber. From our experience of modeling the Non-Proliferation Experiment (which had a similar though less exaggerated tuna can shape) the shock wave lost much of the ellipsoidal effects within $100 \mathrm{~m}$ of the center of the room (Kamm \& Bos 1995).

\subsection{Rubble-Filled Cylindrical Chamber}

One of the standard methods of mining is to rubblize a cylindrical chamber of the material being mined. A room of either tunnel or cylindrical shape is created (as in Section 2.3 above). The miners then systematically work their way upward allowing the roof material to accumulate underfoot, either (1) extracting the material desired and leaving debris behind or (2) leaving all the material for later extraction by various methods (e.g., leeching). This technique provides a very porous type of material that is well known to absorb energy. In this case a rubblized material with $23 \%$ porosity was used from a set of calculations previously reported (Dey \& Bos 1995a) using the effective pressure model in SMC123. This was reported to have a 60 -fold reduction in total energy amplitude in the near source region.

\subsection{Code Descriptions}

SMC123 is a one-, two-, or three-dimensional, non-linear Lagrangian finite-volume finitedifference code (Dey \& Kamm 1994). Specifically designed to model the propagation of impulsive sources of high energy density in geologic material, it has evolved to be able to handle many types of problems in addition to an impulsive source. SMC123 contains one of the most complete suites of material property treatments available in codes of this type. It has been tested successfully against a large set of experimental data.

AFD is an elastic, linear, finite-difference code (Kamm et al. 1996). Its key features are the capability to use arbitrary stratigraphy and arbitrary free surfaces; it also has an anelastic material treatment. It has been tested against problems with analytic solutions with a high degree of accuracy. Linking routines have been developed to map a velocity field from strong motion code simulations as the source for the AFD simulation. 


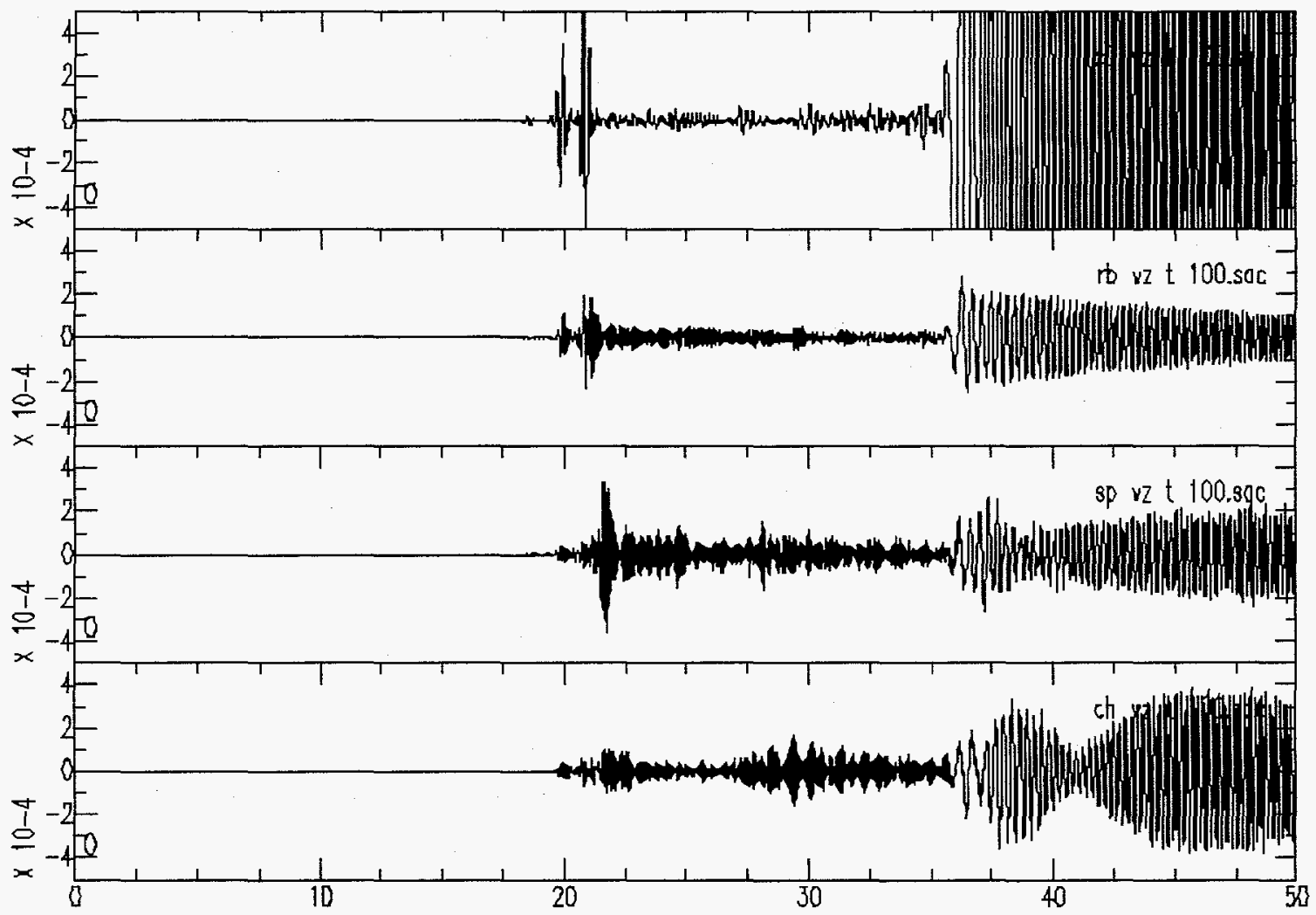

Figure 1

Synthetic waveforms at $100 \mathrm{~km}$ from the source. In order from the top, the individual plots are the tamped, rubble-filled cylindrical chamber, spherical chamber, and mine room chamber. Horizontal axis is time in seconds. Vertical axis is vertical particle velocity in $\mathrm{m} / \mathrm{s}$. See Section 2 of the text for a more detailed description of these simulations.

\subsection{Simulation Results}

Figures 1 and 2 show the synthetic waveforms observed at 100 and $200 \mathrm{~km}$, respectively, for each of the four cases described in Section 2. As noted above, significant ringing occurs with the arrival of the surface wave at $35 \mathrm{~s}$ in Figure 1 and $72 \mathrm{~s}$ in Figure 2. The tamped case waveform at $100 \mathrm{~km}$ in Figure 1 is relatively uncomplicated, comprised of direct $P$ at $18 \mathrm{~s}$, a refracted signal from the second layer at $20 \mathrm{~s}$, and a signal reflected from the second layer at $22 \mathrm{~s}$. No other arrivals of consequence are apparent. The tamped case waveform at $200 \mathrm{~km}$ in Figure 2 is considerably more complicated, comprised of the refracted wave from the mantle at $33 \mathrm{~s}(\mathrm{Pn})$, direct $\mathrm{P}$ at $36.5 \mathrm{~s}$, and the refracted signal from the second layer at $35 \mathrm{~s}$, followed by numerous reflected and refracted additional signals.

As discussed in Section 2, the simulations presented here were expected to have low frequency amplitude ratios between the evasion scenario simulations and the tamped case of $10-100$ to 1 . A visual comparison of the waveforms in Figure 1 shows that the results of these simulations are in contradiction to inferences drawn from previous near source, strong motion evasion study conclusions. The amplitudes of the three evasion scenario simulations are not reduced by the values found in the strong motion code studies but are reduced only by factors of 3-5. These ratios are summarized in Table 2. 
Table 2

Comparison of ratios of peak amplitudes in the P-S part of the waveform of each of the evasion scenarios to the tamped case.

\begin{tabular}{|l|c|}
\hline Rubblized chamber & $3: 1$ \\
\hline Spherical chamber & $3: 1$ \\
\hline Mine chamber & $5: 1$ \\
\hline
\end{tabular}

The decoupling factors determined here have strong implications for evasion scenario detectability. They are different from previous studies primarily for two reasons. The main reason is likely that some stratigraphy is included in these simulations, providing a more complete representation of the entire waveform at near regional distances. Second, the energy of the problem becomes distributed into different parts of the total waveform. Figures 3-6 illustrate these effects in the frequency domain. Note that only the tamped, spherical chamber, and mine room chamber are shown in order to simplify the plots.

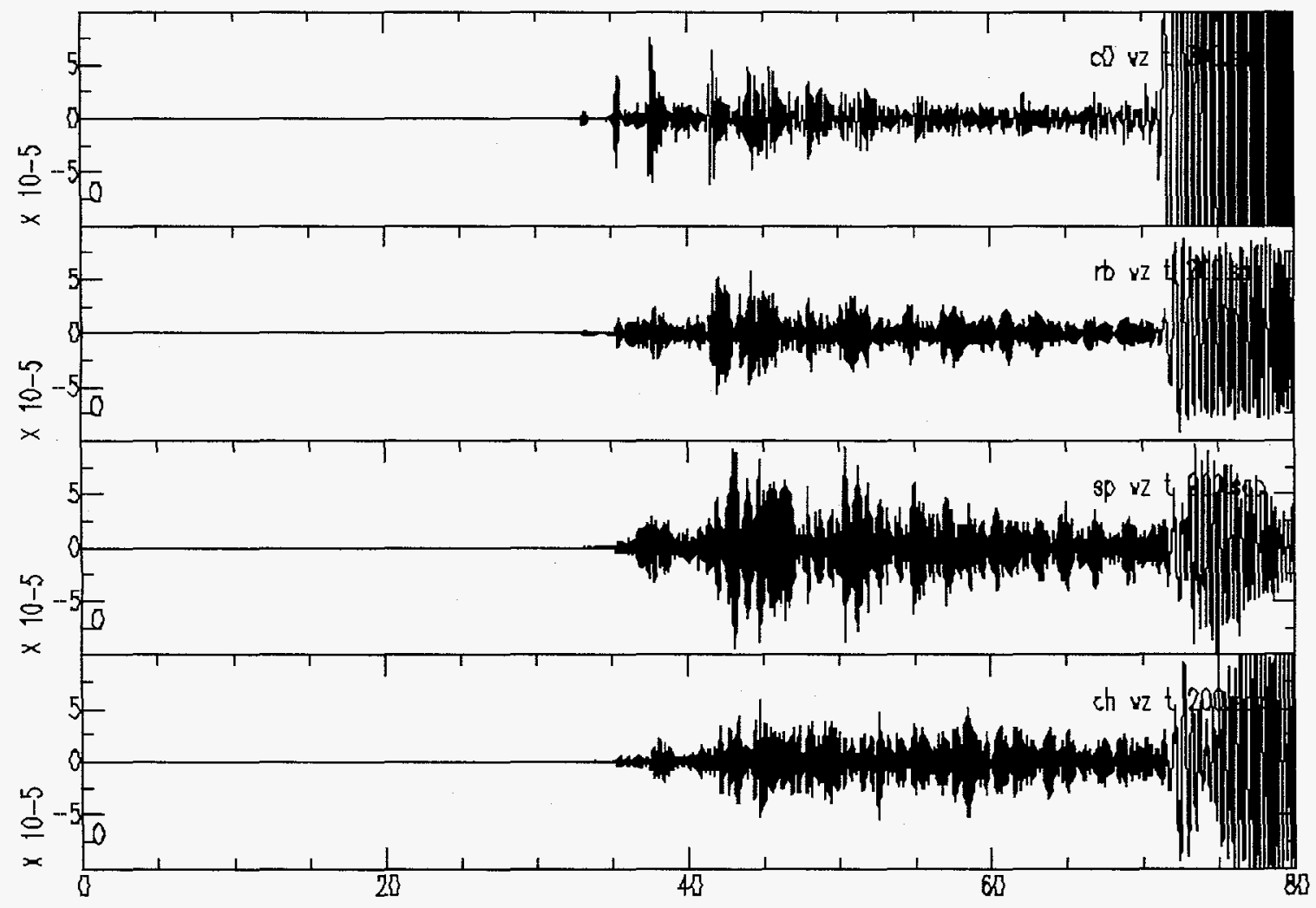

Figure 2

Synthetic waveforms at $200 \mathrm{~km}$ from the source. In order from the top, the individual plots are the tamped, rubble-filled cylindrical chamber, spherical chamber, and mine room chamber. Horizontal axis is time in seconds. Vertical axis is vertical particle velocity in $\mathrm{m} / \mathrm{s}$. See Section 2 of the text for a more detailed description of these simulations. 


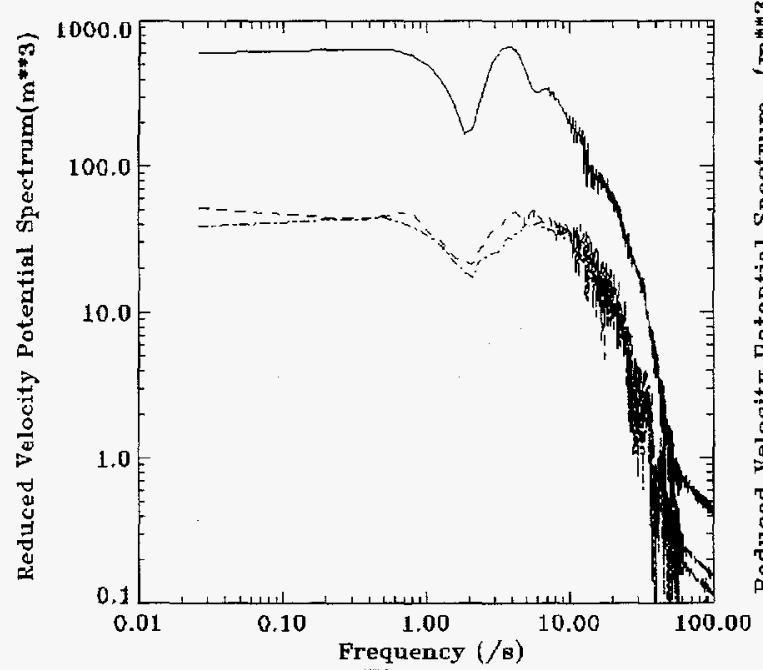

Figure 3

RVP at a location $1 \mathrm{~km}$ from the $1 \mathrm{kt}$ source. SMC123 calculation in a homogeneous medium. The tamped case is the solid line, the spherical chamber is dashed, and the mine chamber is dashed-dot.

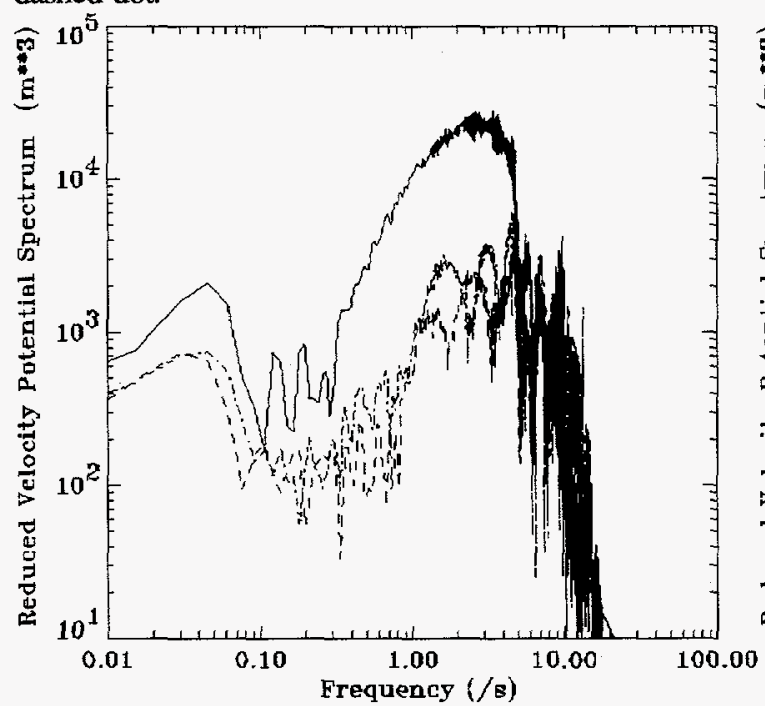

Figure 5

RVP at a location $100 \mathrm{~km}$ from the $1 \mathrm{kt}$ source. AFD calculation in a layered medium using the complete waveform. The tamped case is the solid line, the spherical chamber is dashed, and the mine chamber is dashed-dot.

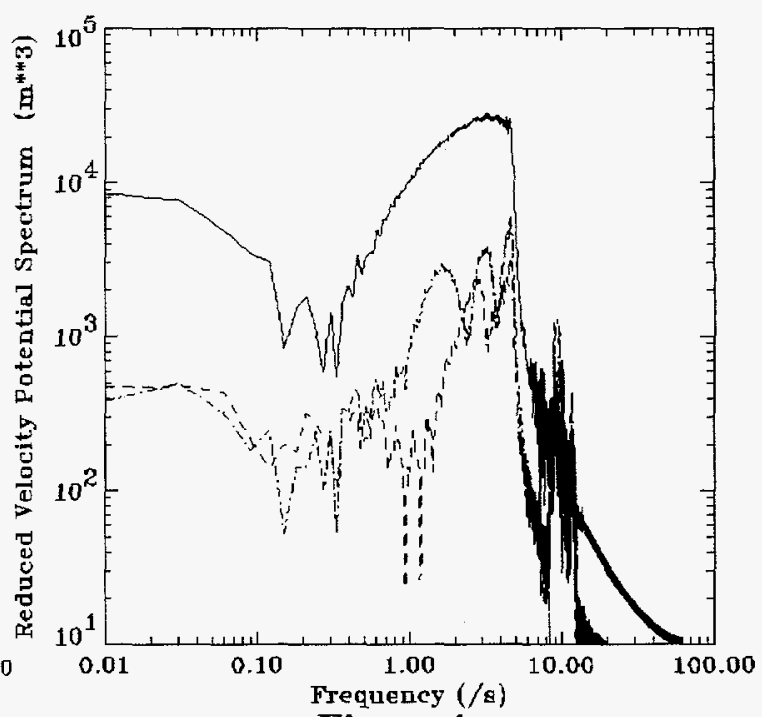

Figure 4

RVP at a location $100 \mathrm{~km}$ from the $1 \mathrm{kt}$ source. AFD calculation in a homogeneous medium. The tamped case is the solid line, the spherical chamber is dashed, and the mine chamber is dashed-dot.

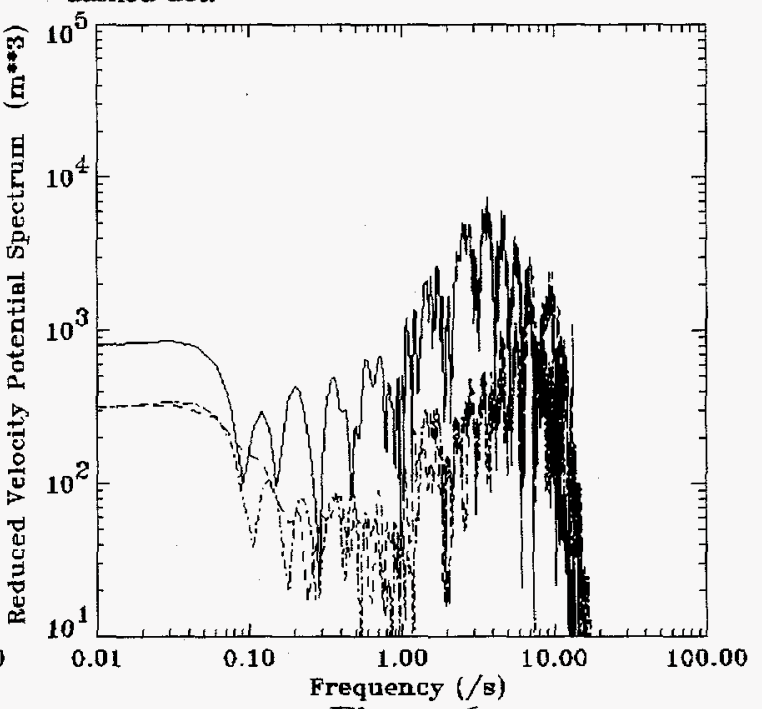

Figure 6

$\mathrm{RVP}$ at a location $100 \mathrm{~km}$ from the $1 \mathrm{kt}$ source AFD calculation as in Figure 5 but only using the $\mathrm{P} / \mathrm{S}$ part of the waveform. The tamped case is the solid line, the spherical chamber is dashed, and the mine chamber is dashed-dot.

The evolution of energy partitioning with increasing distance from the source can be seen in a comparison of Figures 3 and 4. Figure 3 shows the RVP computed at $1 \mathrm{~km}$ from the source, and Figure 4 shows the RVP computed at $100 \mathrm{~km}$ from the source. Both calculations are done in a homogeneous medium, so the only effect present is the partitioning of energy into the various components of the waveform (e.g., P, S, and R phases). Also observe in Figures 3 and 4 that the decoupling factors are roughly the same between the SMC123 calculation and the AFD calculation done in a homogeneous medium 
(the enhanced higher frequency bump in Figure 4 is partially due to the numerical ringing described above). In direct comparison to Figure 4, Figure 5 shows the effect of including a layered medium in the simulation. The decoupling factors are now consistent with the amplitude ratios given in Table 2 . The frequency spectrum has also shifted to the higher frequencies (considering that the calculation is only supporting up to $3-4 \mathrm{~Hz}$ ).

As described above, the numerical ringing will contribute to the RVP. Using the same waveforms as were used to calculate the RVPs shown in Figure 5 but cutting off the surface wave component above $35 \mathrm{~s}$ (see Figure 1) produces the RVPs shown in Figure 6. The low frequency component ratio of the two evasion scenario RVPs to the tamped case RVP is roughly the same as the amplitude ratios listed in Table 2.

It is clear from these results that, at least for the cases presented here, using near source waveforms before the waveform has had time to fully develop may lead to misleading extrapolations at regional distances for any particular evasion scenario. In addition, using either the relative amplitudes of the synthetic seismogram or RVPs generated from those seismograms at regional distances without accounting for layering may lead to misleading results regarding the decoupling efficiency of a particular evasion scenario.

Next we address the issue of potential $P$ to $S$ signal modification for the three decoupling scenarios. Returning to Figures 1 and 2, we observe that the ratio of early to late arriving energy ( $\mathrm{P}$ to $\mathrm{Lg}$ ) for the spherical cavity and rubblized chimney cases is about the same as for the tamped case. However, the overall frequency content is higher for the decoupled cases. At present it is not clear if this is due to a real effect from an extended source or an enhancement of higher frequencies due to the linking between SMC123 and AFD. There is no obvious strong Lg component for either the spherical cavity or the rubble case (the earliest portion of a shear signal should be arriving at about $32 \mathrm{~s}$ at $100 \mathrm{~km}$ and $60 \mathrm{~s}$ at 200 $\mathrm{km})$. The signal for the mine chamber is somewhat different from the other three cases and we will discuss this further below. We conclude that, under the assumptions made in these calculations, the spherical cavity and rubblized chamber cases have a definite explosive appearance.

As noted above, the primary difference between the mine chamber case and the other simulations is the signal between 26 and $32 \mathrm{~s}$ at $100 \mathrm{~km}$. Although not as evident, it is clear there is again some additional signal at $200 \mathrm{~km}$ as well. The only difference between this and the other two evasion scenarios is the source geometry as described in Section 2 . Figures 7-10 are SMC123 particle velocity vector plots for the near source region for the spherical chamber and mine chamber cases. Comparison of Figures 7 and 8 , at $75 \mathrm{~ms}$ for the two cases, indicates there are some potentially significant differences. A shear wave has developed at a radius of $150 \mathrm{~m}$ from the source at $-300 \mathrm{~m}$ in the mine chamber plot (Figure 8). This wave can be seen to be comprised of two vortical structures, the one at $-400 \mathrm{~m}$ being the most clearly defined. This feature is not present in the spherical chamber case. Figures 9 and 10 again compare the two cases but at $125 \mathrm{~ms}$.

Now the shear band in the mine chamber scenario is interacting with the surface. There is also a clear interaction of this shear band with the $\mathrm{pS}$ wave coming off the surface. Again this complex developing wave structure is not seen in the spherical chamber scenario in Figure 9. Following this wave back in time (plots not shown), it is seen that the source energy propagates up and down from the flat surfaces of the top and bottom of the 


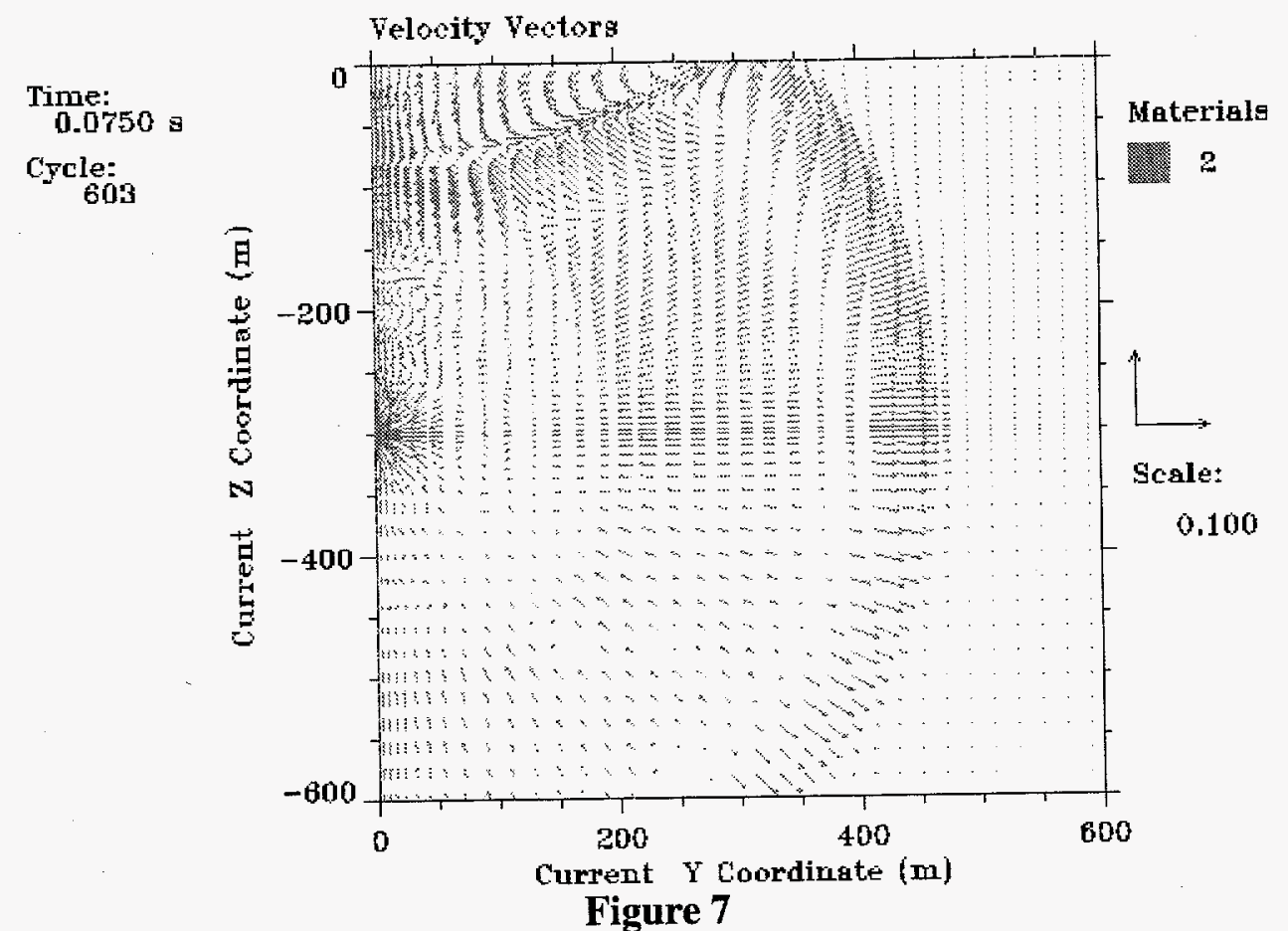

Particle velocity field at $75 \mathrm{~ms}$ for the spherical chamber scenario described in the text.

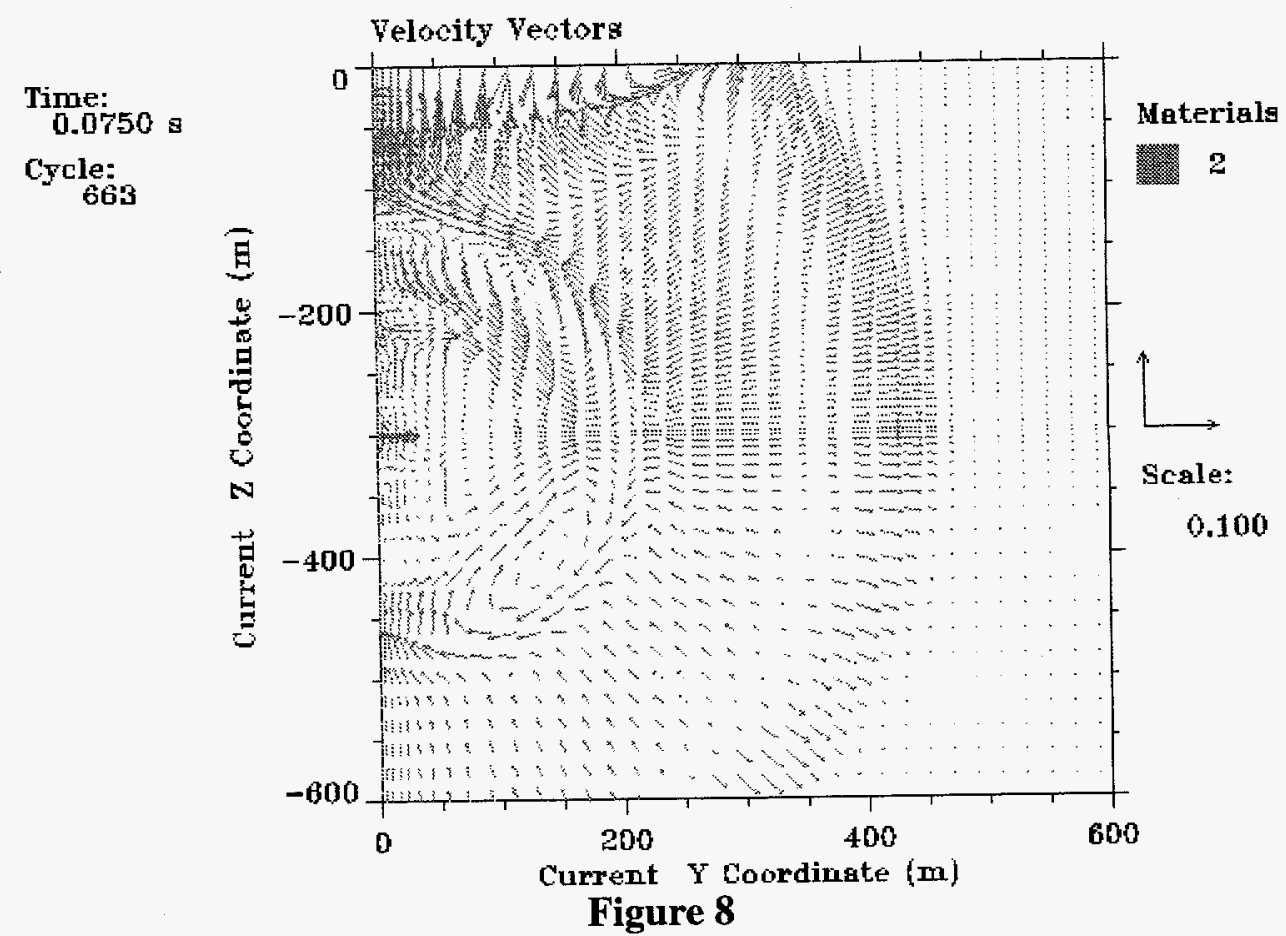

Particle velocity field at $75 \mathrm{~ms}$ for the mine chamber scenario described in the text. Note the shear waves present here but not in Figure 7. 
Velocity Vectors

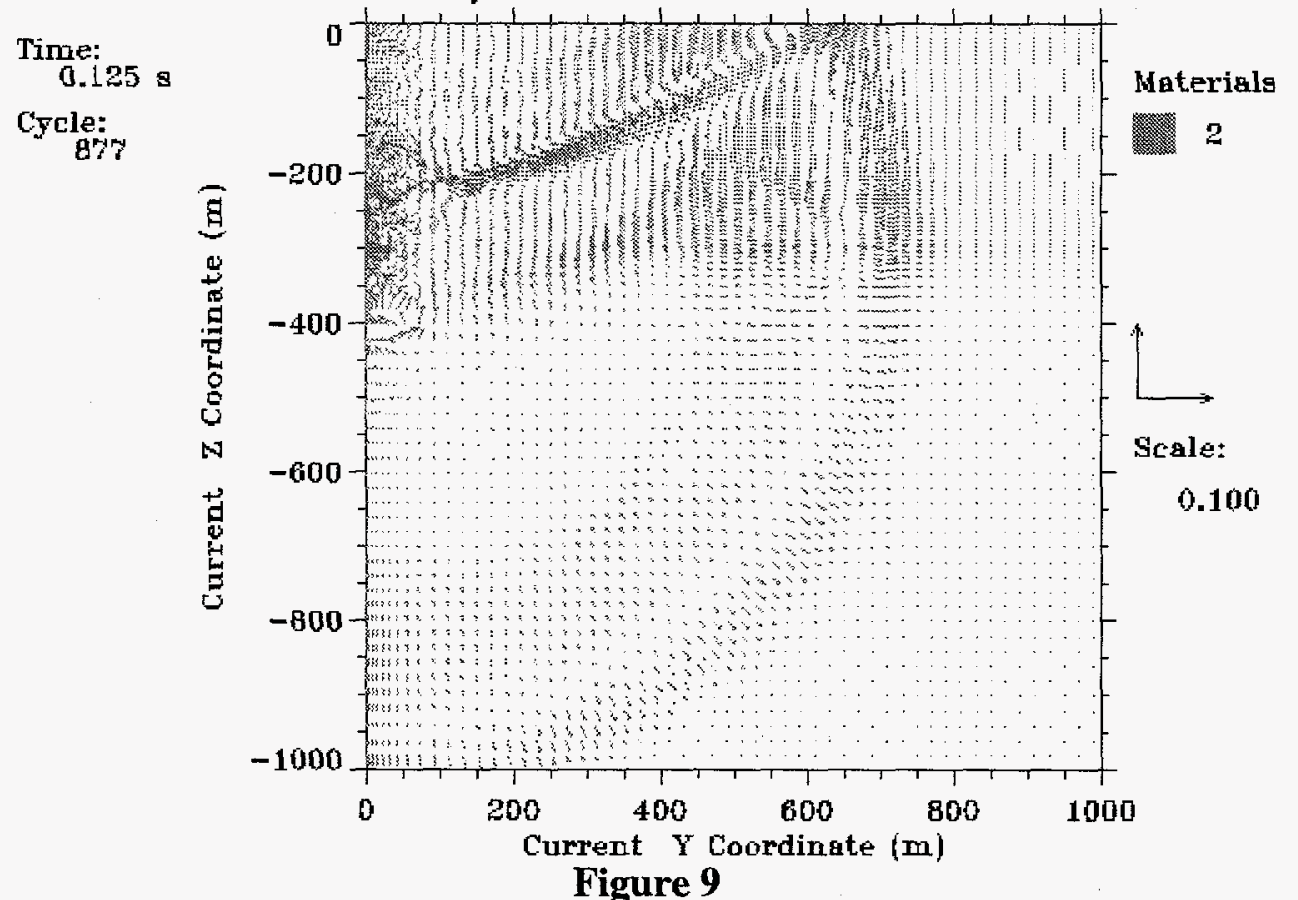

Particle velocity field at $125 \mathrm{~ms}$ for the spherical chamber scenario described in the text. Same simulation as Figure 7 except at a later time.

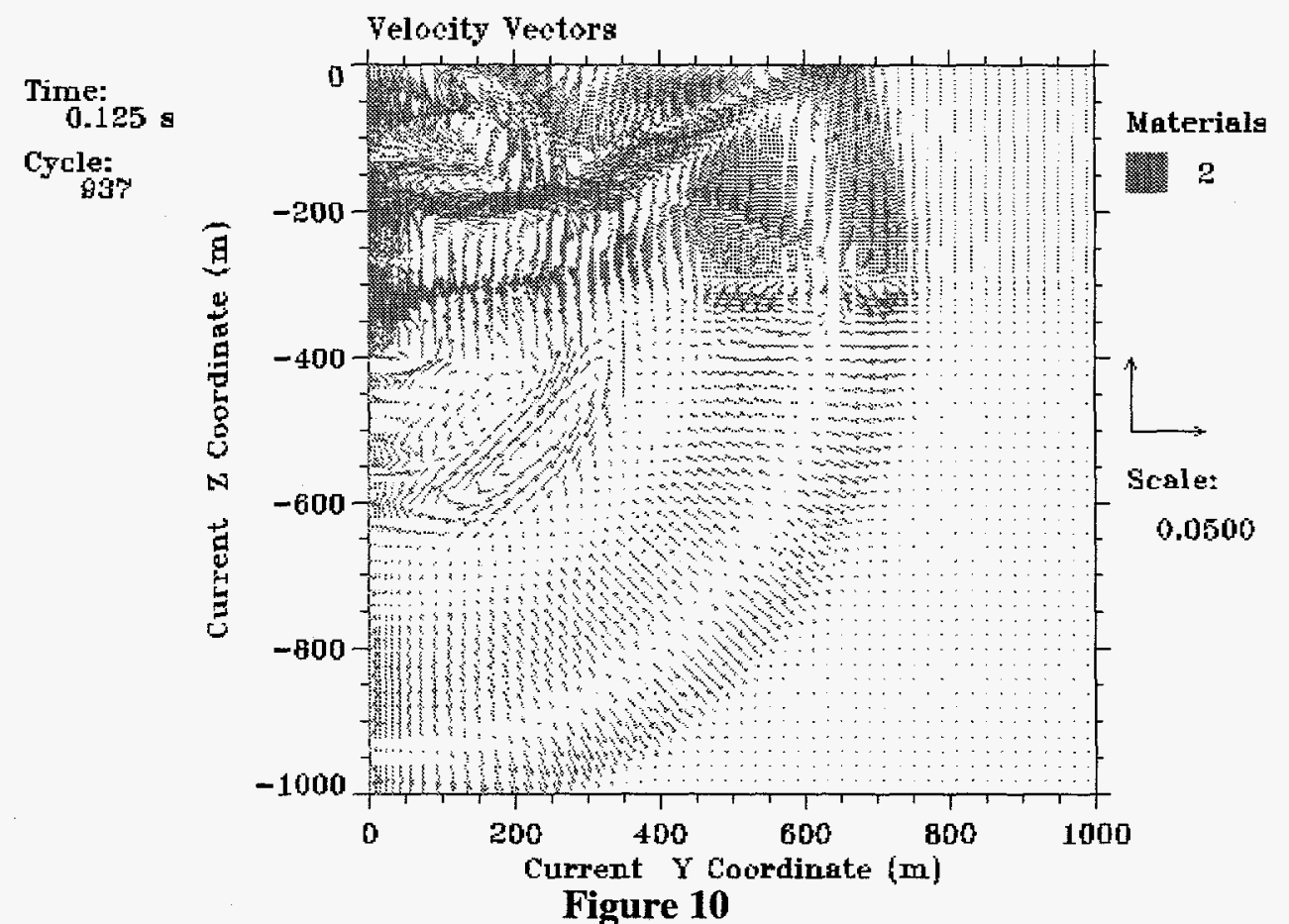

Particle velocity field at $125 \mathrm{~ms}$ for the mine chamber scenario described in the text. Again note the shear waves present here but not in Figure 9. 
chamber, respectively. At the edges of the chamber, the velocity field begins to curl around, producing a toroidal velocity field that develops into the shear band seen in Figures 8 and 10. Following this band out to the time of Figures 1 and 2 and observing the different reflections it goes through allows us to conclude that this is what gives rise to the increased amplitude of the signal at $26-32 \mathrm{~s}$ at $100 \mathrm{~km}$ for the mine chamber case. An explosion in the mine chamber configuration results in a larger proportion of shear to compressional energy than in the other cases; therefore, this case may be deserving of further attention in various evasion scenarios.

\subsection{Discussion}

The results indicating regional detectability for the suite of evasion scenarios studied for this work were quite surprising to us given the previous work of some of the authors (Dey $\&$ Bos 1995b). The assumption that the relatively high decoupling ratios inferred by comparing the low frequency spectral response characteristics of various scenarios calculated from near source simulations may not be valid at regional distances if realistic path effects, such as stratigraphic layering, are not taken into account. In order to make plausible estimates of the effect of various evasion scenarios from simulations, one must include realistic descriptions of stratigraphy and perhaps topography. This is especially true if one is studying a specific region.

The other important result from a discrimination point of view is that the signals from two of the evasion scenarios retain their explosive characteristics at regional distance irrespective of the amplitude question. The results of the chamber evasion scenario indicate that there may be some source geometries that could lead to more earthquake-like seismic signals.

\subsection{References}

Dey, T. N., "Influence of Equation of State and Constitutive Behavior on Seismic Coupling," in Proceedings of the Numerical Modeling for Underground Nuclear Test Monitoring Symposium, Los Alamos National Laboratory document LA-UR-93-3839, pp. 157-168, 1993.

Dey, T. N., and R. J. Bos, "Decoupling Nuclear Explosions in Rubble Filled Cavities," in Proceedings of the Nuclear Testing Evasion Symposium, Defense Nuclear Agency, 1995a.

Dey, T. N., and R. J. Bos, "Potential Evasion Scenarios for Central Asia (U)," Los Alamos National Laboratory report LA-12987-MS (S-NSI), September 1995b.

Dey, T. N., and J. R. Kamm, "User's Guide to SMC-2D v3.1," Los Alamos National Laboratory document, 1994.

Glenn, L. A., "Energy Density Effects on Seismic Decoupling," J. Geophys. Res., 98, pp. 101-109, 1993.

Glenn, L. A., "Kuchen: An Experiment to Evaluate Decoupling in High Aspect Ratio Cavities," in Proceedings of the Nuclear Testing Evasion Symposium, Defense Nuclear Agency, 1995. 
Kamm, J. R., and R. J. Bos, "Comparison of Chemical and Nuclear Explosions: Numerical Simulations of the Non-Proliferation Experiment," Los Alamos National Laboratory report LA-12942-MS, June 1995.

Kamm, J. R., R. J. Bos, and E. M. Jones, "User's Guide to AFD v. 1.0," Los Alamos National Laboratory document LA-UR-96-853, March 1996.

Latter, A. L., R. E. LeLevier, E. A. Martinelli, and W. G. McMillan, "A Method of Concealing Underground Nuclear Explosions," J. Geophys. Res., 66, pp. 943-946, 1961.

Murphy, J. R., and B. W. Barker, "Seismic Identification Analyses of Cavity Decoupled Nuclear and Chemical Explosions," Phillips Laboratory report PL-TR-94-2036, Jan. 1994.

Murphy, J. R., I. O. Kitov, J. L. Stevens, D. D. Sultanov, B. W. Barker, N. Rimer, and M. C. Friedman, "Analyses of the Seismic Characteristics of U.S. and Russian Cavity Decoupled Explosions," Phillips Laboratory report PL-TR-94-2295, Nov. 1994.

Reinke, R., "High Explosive Decoupling Experiments in Hard Rock," in Proceedings of the Nuclear Testing Evasion Symposium, Defense Nuclear Agency, 1995.

Spivak, A. A., and V. V. Adushkin, "Study and Analysis of Experiences of Explosive Development and Construction for Underground Tests (U)," Defense Nuclear Agency technical report, Contract DNA001-95-C-0027 (Protect as Confidential), 1995.

Springer, D., M. Denny, J. Healy, and W. Mickey, "The Sterling Experiment: Decoupling of Seismic Waves by a Shot-Generated Cavity," J. Geophys. Res., 73, pp. 5995-6011, 1968.

Stevens, J. L., J. R. Murphy, and N. Rimer, "Seismic Source Characteristics of Cavity Decoupled Explosions in Salt and Tuff," Bull. Seis. Soc. Am., 81, pp. 1272-1291, 1991.

Stevens, J. L., T. G. Barker, E. J. Halda, J. R. Murphy, and N. Rimer, "Simulation of Teleseismic Body Waves from Underground Explosions Beneath Sloping and Flat Surfaces Using Nonlinear Axisymmetric Finite Difference Calculations," S-Cubed Technical Report to the Defense Nuclear Agency, DNA-TR-91-5, December 1990.

Terhune, R. W., C. M. Snell, and H. C. Rodean, "Enhanced Coupling and Decoupling of Underground Nuclear Explosions," Lawrence Livermore Laboratory report UCRL-52806, 1979. 